\title{
Air Speed Indication Calibration Measurement Position Error Correction - PEC
}

\author{
O. Freund
}

\author{
Airbus Helicopters Germany
}

Otto.Freund@airbus.com

Key words: Airspeed, Altitude, Indication, Position error correction, trailing bomb, pressure scanner.

\section{Position Error determination with new tools}

The airspeed and altimeter systems on an aircraft depend upon accurate measurements of ambient static pressure and total pitot pressure.

Static and pitot pressures are sensed by the pitot static tube, which gives true readings in an undisturbed free stream when aligned with the flow streamlines; however, when attached to the aircraft, which generates a pressure when flying, the pitot and the static reading will be affected by the aircraft pressure field and the flow angularity.

The errors, caused by the pressure field and by flow angularity are called position errors due to the sign and magnitude of the errors, which are a function of the position of the pitot-static probe on the aircraft.

The position errors are a function of aircraft angle of attack and Mach number and are determined from flight test.

This presentation shows one way of establishing these errors using a trailing bomb which measures static and dynamic pressure as well as a miniature electronic differential pressure scanner using a new method to quickly establish results.

\section{Old versus New}

\section{Past}

In the past the PEC flight test technique used circular differential pressure indicating gauges, a static pressure bomb or cone and a nose boom for total pressure as well as side slip angle.

- The gauges have systematic errors due to the design and are difficult in use, as the indicator needle is always fluctuating during flight.

They need to be calibrated before the use, considering the hysteresis and friction.

- The difference in length of the sensing lines can introduce errors.

- Only limited use for climb and descent Lag Error

- The analysis is time consuming as all the calibrations have to be included.

- The tests are very time consuming as every flight condition needs to be stabilized before the readings can be taken.

\section{Present}

The availability of new trailing bombs as well as measurement equipment lead to a new method.

The primary advantage, next to accuracy, is the continuous data, allowing a multitude of analysis. 
Because of the availability of continuous data a new method for determining the air speed and altitude indication error for different pitch attitudes was tested with good results.

\section{Test preparation}

The trailing bomb, which provides static as well as total pressure, was connected to the helicopter with a steel cable which was fitted with two weights to provide proper separation in all flight conditions. Their correct position was determined in flight. Even small changes in the position of the lower mass had substantial influence on the stability of the trailing bomb. The separation is shown in Figure 1.

The tubes were run along the steel cable and connected to the pressure scanner on board. The cable with the tubes was covered with a plastic mesh to reduce the load on the tubes and to provide better aerodynamics.

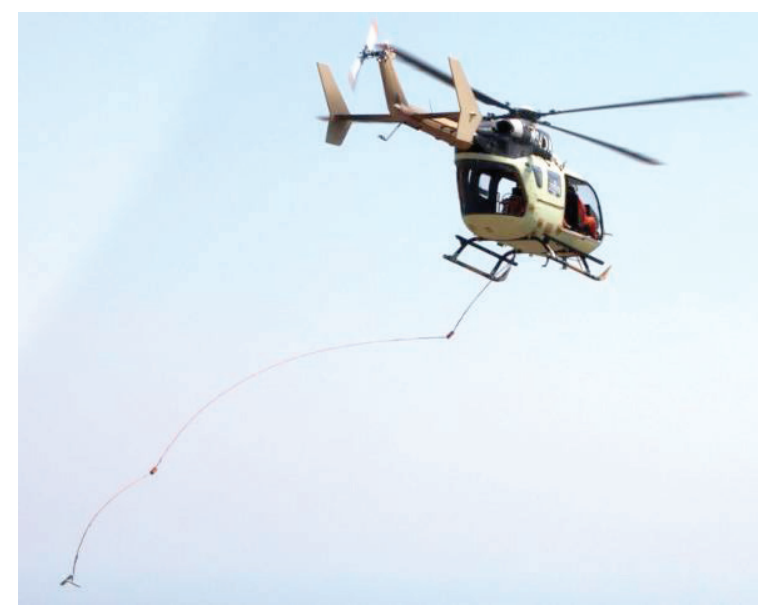

Figure 1: Trailing Bomb: Final Configuration, Descent 100 kts, $1500 \mathrm{fpm}$

\section{Measurement Setup}

Static and dynamic pressures for the PEC flights are routed to a 16-channel electronic pressure scanner with temperature compensation (Figure 2).

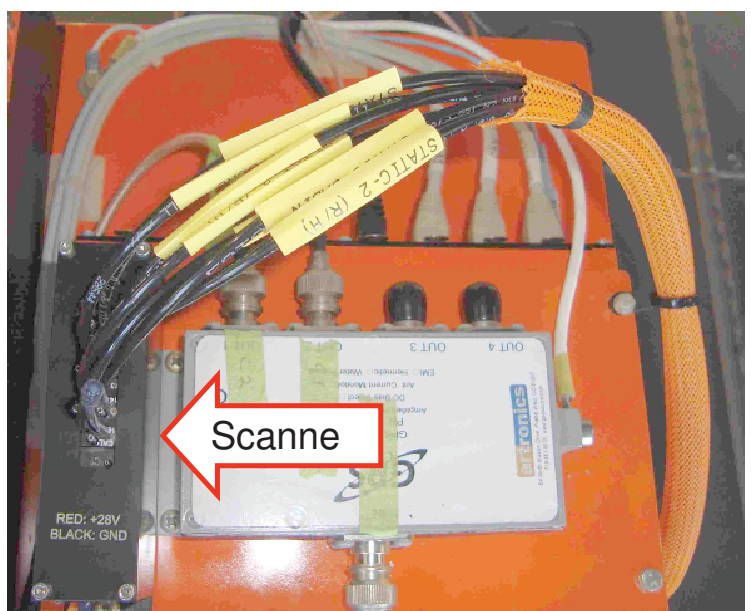

Fig.2 Pressure Scanner in heating chamber

All pressures are referenced to a common pressure, which was chosen to be the trailing bomb reference static pressure.

Helicopter PEC takes place in the lower airspeed spectrum of low static and dynamic pressures. Using a common differential reference minimizes the effects of sensor error and non-optimal stabilization of airspeed/altitude.

The yellow block represents the steps required to derive the installation errors. The reference pressures from the trailing bomb are assumed to have no errors, measuring free air static and pitot pressure perfectly. Also, the errors from the pressure sensor are within $\pm 0.15 \mathrm{mb}$ and are verified using pre- and post-flight calibrations.

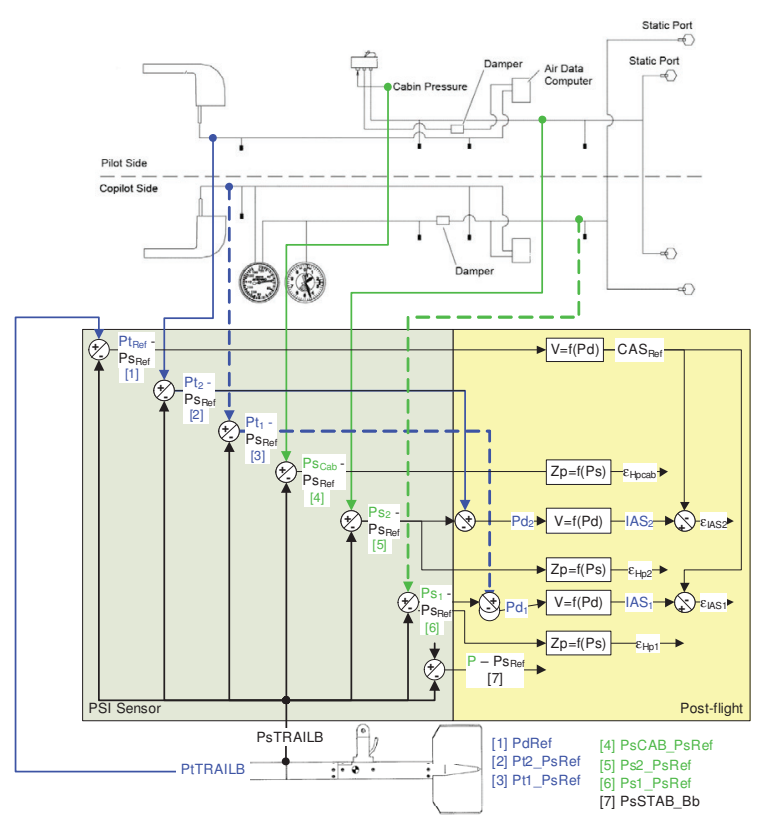

Fig. 1 Measurement Setup and calculation 
The measurement chain errors are considered to be well within the scatter resulting from nonperfect stabilizations.

PEC pressure error is converted to altitude and airspeed error using only two equations:

$$
\begin{gathered}
\varepsilon_{H p[f t]}=-27 \Delta P_{S[m b]}(1) \\
V_{[k t]}=661.3\left\{\frac{1}{0.2}\left[\left(\frac{P_{d[m b]}}{1013.25}+1\right)^{\frac{1}{3.5}}\right]-1\right\}^{\frac{1}{2}}
\end{gathered}
$$

This allowed calculating all required parameters as well as displaying the results directly to the Flight Test Engineer on board.

\section{PEC Flight Test Technique (FTT)}

A simple Quasi-Static test method was considered to minimize the flight time and enhance safety. While the traditional method is based on steady-state measurements, the Quasi-Static FTT consists of performing a slow airspeed sweep $(\sim 1 \mathrm{kts} / \mathrm{sec})$ and continuously recording pitot-static data. In the data reduction phase, a stability criteria is applied, locating stable time periods in which the averaged data is calculated. By sweeping the airspeed in both directions, any hysteresis effect is brought to evidence or attributed to a secondary parameter (for example, aircraft pitch attitude).

For helicopters, the effect of airspeed and CG on pitch attitude is approximated by a linear function, as shown in Figure 3:

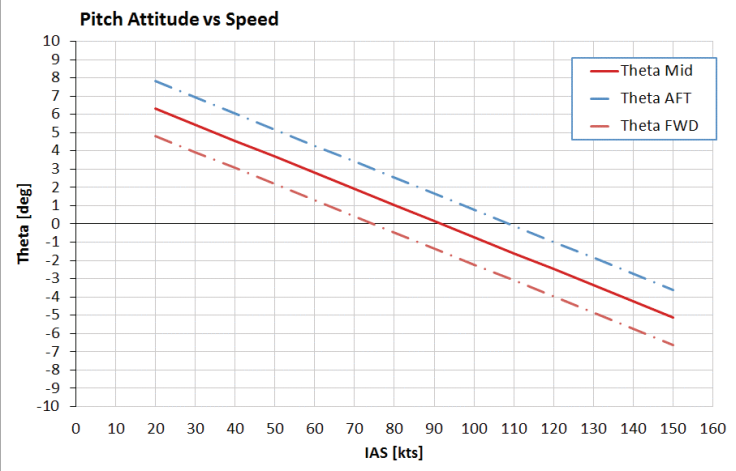

Fig. 3 Pitch attitude versus airspeed for different center of gravities

An onboard dynamic display, providing a realtime depiction of the last 10 seconds of Pitch Attitude vs. IAS, was prepared for the pilot to execute in flight. A depiction of this dynamic display is shown in Figure 4

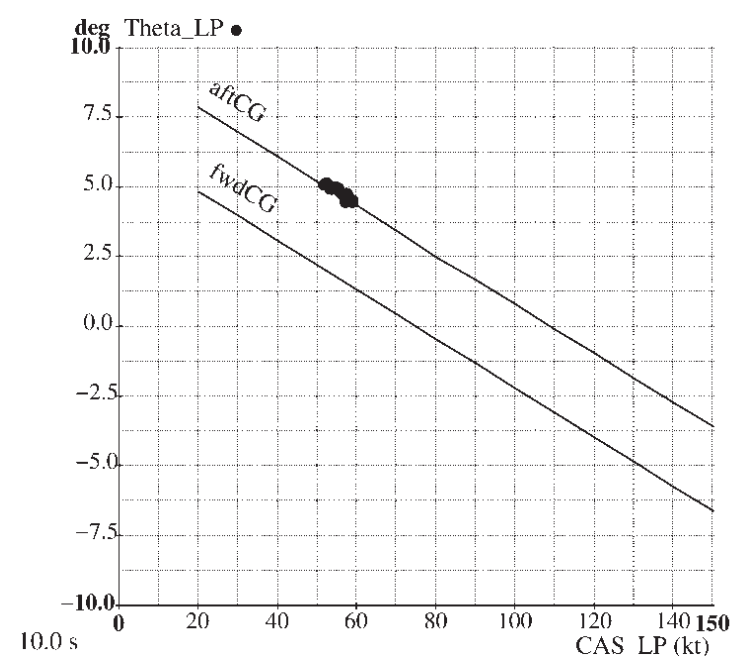

Fig. 4 On-Board Dynamic Pilot Display

\section{Test Execution}

Prior to and after each test the Zero reading of the pressure sensors was taken to allow an offset correction of the measured parameters.

Steady state measurements were conducted with 10 to 20 seconds of stabilized data for reference purposes. For the Quasi-Static FTT, the airspeed change rate required to fly the attitude line was 1 to 2 knots per second. The time needed to complete each sweep required approximately 2 minutes.

\section{Results}

The PEC test results are shown in Figure 5 and Figure 6 for the altitude and airspeed errors respectively. The red test points represent steady state measurements, with triangles and stars showing quasi-static test points for acceleration and deceleration, respectively.

Deceleration data is identical with the steady state data, indicating no change in PEC from mid to forward $C G$ range.

Acceleration data is significantly different in the low airspeed range and is traced to the static port installation being affected by a shift of the neutral static pressure boundary from 20 to 60 KIAS. This characteristic was not revealed by steady-state measurements.

Another benefit of the Quasi-Static FTT method is the large amount of data produced in very short test time. Resulting graphs are very densely populated with a wealth of information, including the stability of the measurements and possible local phenomena. 


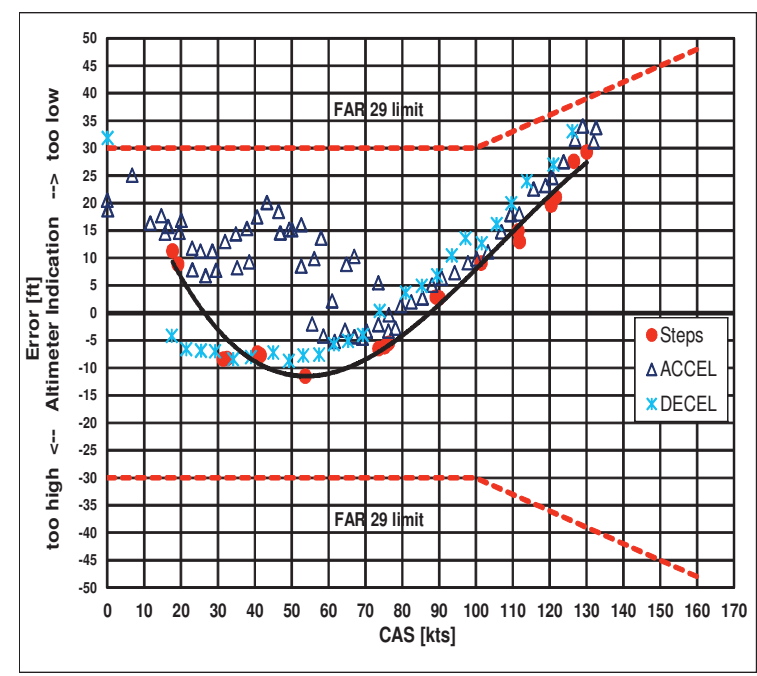

Fig. 5 Altitude PEC

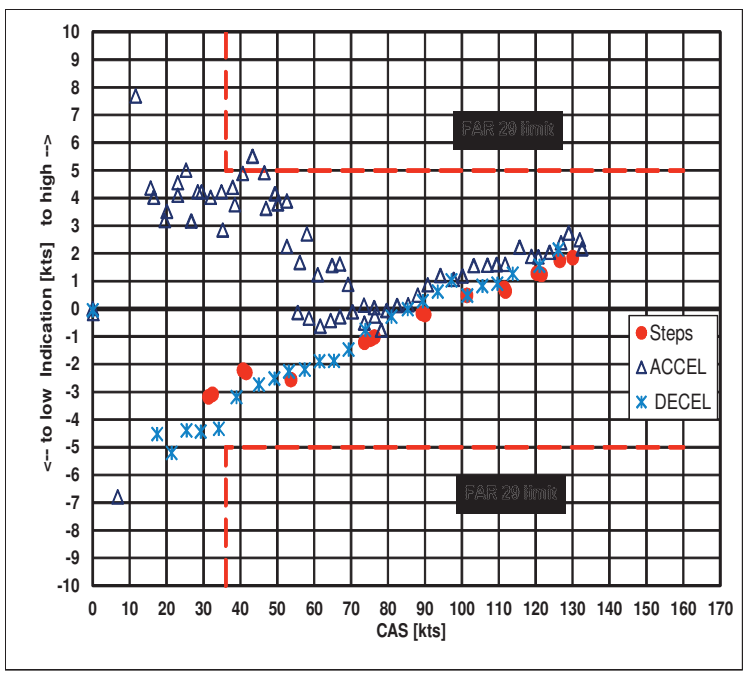

Fig. 6 Airspeed PEC

\section{Lessons Learned}

Special attention with the FTI calibration was necessary to ensure that the required accuracies were achieved. In particular, pressure sensors were extremely sensitive to temperature variations. The FTI calibration had to account for this variable, so that additional errors were not introduced into the test results.

The Quasi-static FTT for conducting PEC flights provides an extremely efficient means of gathering position-error data for three CG extremes in a single sortie. The benefits to flight test are profound: since fewer test sorties and few configuration changes are required (with no need to re-ballast the aircraft), the impact on safety is equally significant.

The Quasi-Static FTT meets the accuracy requirements for $\mathrm{PEC}$ testing during level flight. Further evaluation and analysis is needed to extend the technique to climbing and descending flight.
The use of Chase aircraft was essential to the development of an acceptable trailing bomb configuration. Since "acceptable" behavior of the trailing bomb was essential throughout an airspeed range from hover to $1.1^{*} \mathrm{VNE}$, experienced flight test crewmembers were required to man the Chase aircraft. This provided for accurate characterization of the trailing bomb flight behavior, in addition to allowing for ATC communications, airspace management, and efficient test sequencing. 